\section{CPS-240 A QUALITATIVE STUDY ON HOW CLINICAL PHARMACISTS PREFORM MEDICATION RECONCILIATION IN THE EMERGENCY DEPARTMENT}

${ }^{1,2} \mathrm{MK}$ Moan Ingebrigtsen*, ${ }^{2,3} \mathrm{~J}$ Kutschera Sund, ${ }^{1} \mathrm{M}$ Winge, ${ }^{4} \mathrm{HT}$ Bell. 'Levanger Hospital Pharmacy, Central Norway Hospital Pharmacy Trust, Levanger, Norway; ${ }^{2}$ Faculty of Medicine and Health Sciences, Norwegian University of Science and Technology, Trondheim, Norway; ${ }^{3}$ Central Norway Hospital Pharmacy Trust, Central Norway Hospital Pharmacy Trust, Trondheim, Norway; ${ }^{4}$ Faculty of Nursing and Health Sciences, Nord University, Levanger, Norway

10.1136/ejhpharm-2019-eahpconf.389

Background An accurate drug history is an essential part of patient assessment at admission to hospital. Studies show that pharmacists obtain a more accurate medication history than other health professionals. In many countries clinical pharmacists work in the emergency department (ED) performing medication reconciliation (MR). Although many quantitative studies describe the effect of clinical pharmacists in the ED, to our knowledge, there are no qualitative studies on how clinical pharmacists preform MR and which factors they perceive to affect their work.

Purpose The aim of this study was to describe how clinical pharmacists perform $\mathrm{MR}$ in an ED and to identify barriers and factors influencing all steps of MR such as preparation, patient interview and documentation.

Material and methods The study was conducted in the ED in a hospital with 173 beds. A non-participating observational method was used and a standardised observation form was developed based on existing procedures. Seven hospital pharmacists were included and $61 \mathrm{MR}$ were observed over 10 days. Based on the findings from the observation study, a semi-structured focus group interview with five hospital pharmacists was conducted. Data from the observation study was described in relation to the existing procedure, and together with data from the interview, analysed using Systematic Text Condensation.

Results Variations were observed and influencing factors identified and organised in three themes: the patient, the clinical pharmacist and the workflow in the ED.

The complexity of the patient's medication history affected how the pharmacists prepared for, and conducted, the interview. The patients' relatives and the general condition of the patient also had an impact on the questions asked.

The degree of clinical experience and training influenced the clinical pharmacists' decisions in all phases of the MR, as well as the clinical pharmacists' assertiveness.

The clinical pharmacy service was not fully integrated in the ED workflow, and although the clinical pharmacists felt integrated, they seemed to perform their service in parallel with other healthcare professionals.

Conclusion Several factors have an impact on how clinical pharmacists conducts $\mathrm{MR}$ in an $\mathrm{ED}$ and influence their choices. This study shows that the service provided by the clinical pharmacists are not optimal and should be further developed.

\section{REFERENCES AND/OR ACKNOWLEDGEMENTS}

No conflict of interest.
4CPS-241 DDI-PREDICTOR: A NOVEL CLINICAL PHARMACY DECISION-MAKING TOOL FOR DOSE ADAPTATION?

${ }^{1} \mathrm{~F}$ Moreau*, ${ }^{1,2} \mathrm{~N}$ Simon, ${ }^{3,4} \mathrm{M}$ Tod, ${ }^{1,2} \mathrm{~B}$ Decaudin, ${ }^{1,2} \mathrm{P}$ Odou. ${ }^{1} \mathrm{CHU}$ Lille, institut de pharmacie, lille, france; ${ }^{2}$ Univ. Lille, EA 7365 - Grita - Groupe de Recherche sur les Formes Injectables et les Technologies Associées, Lille, France; ${ }^{3}$ Université Lyon 1-Faculté de Médicine Lyon-Sud-Charles Mérieux, Emr 3738, Oullins, France; ${ }^{4}$ Groupement Hospitalier Nord- Hospices Civils de Lyon, Pharmacy, Lyon, France

\subsection{6/ejhpharm-2019-eahpconf.390}

Background To date, pharmacists have been limited to advising physicians about changes in drug prescriptions in the case of drug-drug interactions (DDI), cirrhosis or the presence of genetic polymorphism on $\mathrm{P} 450$ cytochromes (CYP). Dose adaptation is complicated. DDI-Predictor (DDI-P) is a free online application composed of five modules. Three modules are: drug-drug interaction; drug exposure level in case of cirrhosis; and drug exposure level in case of genetic polymorphism for CYP2D6, 2 C9 and 2 C19. The other two modules are combinations of the previous three modules, namely (a) $+($ b) or $(a)+(c)$.

Purpose To describe DDI-P use as a clinical pharmacy decision-making tool.

Material and methods Eighteen clinical pharmacists were trained before using DDI-P. DDI-P computed a ratio of area under the drug-concentration curves $\left(\mathrm{R}_{\mathrm{AUC}}\right)$ by comparing an AUC to a standard. Dose adaptation was calculated from $\mathrm{R}_{\mathrm{AUC}}$. Pharmaceutical intervention (PI) was advised if $0.5 \leq \mathrm{R}_{\mathrm{AUC}}$ (induction) or $\mathrm{R}_{\mathrm{AUC}} \geq 1.5$ (inhibition). Data recorded in a standardised datasheet in Excel software (Microsoft, France) were: date, drug and posology, interacting drug, cirrhosis grade, module used, $\mathrm{R}_{\mathrm{AUC}}, \mathrm{PI}$ and medical acceptation (MA). Data were analysed by one referent pharmacist. The endpoints were pharmaceutical intervention and medical acceptation rates.

Results 199733 prescriptions were analysed during 26 months and 290 cases involved DDI-P. Seventy-seven cases were excluded (infructuous research, $n=43$; application misuse, $n=30$; uninterpretable results, $n=4)$. Other cases concerned DDI with inducers $(n=56 ; 26 \%)$ or inhibitors $(n=145 ; 68 \%)$ and cirrhotic patients $(n=12)$. PI occurred in 121 cases $(56.8 \%)$, for inducers $(75 \%)$, for inhibitors $(54 \%)$ and for cirrhosis (66\%). For inducers with $0.5 \leq \mathrm{R}_{\mathrm{AUC}}$, PI concerned: drug switch (33\%) and interactor stop (6\%). For inhibitors with $\mathrm{R}_{\mathrm{AUC}} \geq 1.5$, PI were dose-lowering (17/79) or drug switch (7/79). The MA rates were $88 \%$ and $82 \%$ for inducers and inhibitors, and $100 \%$ for cirrhosis, respectively.

Conclusion This first study assessing DDI-P shows how it may help clinical pharmacists in their daily practice. $\mathrm{R}_{\mathrm{AUC}}$ value leads pharmacists to assess the importance of DDI and to propose therapeutic adjustments to physicians, contributing to therapeutic decisions. Although it is easy to use, pharmacists must therefore be trained to interpret the result in the clinical context at the time of the analysis to avoid potential misuses.

REFERENCES AND/OR ACKNOWLEDGEMENTS

No conflict of interest. 